Pacific

Journal of

Mathematics

\title{
HIGHER-ORDER INVARIANTS OF IMMERSIONS OF SURFACES INTO 3-SPACE
}

TAHL NOWIK

Volume $223 \quad$ No. 2

February 2006 


\title{
HIGHER-ORDER INVARIANTS OF IMMERSIONS OF SURFACES INTO 3-SPACE
}

\author{
TAHL NOWIK
}

\begin{abstract}
We classify all finite-order invariants of immersions of a closed orientable surface into $\mathbb{R}^{3}$, with values in any abelian group. We show that they are all functions of an order one invariant.
\end{abstract}

\section{Introduction}

Finite-order invariants of stable immersions of a closed orientable surface into $\mathbb{R}^{3}$ were defined in [Nowik 2004], and all order-1 invariants were classified there. Explicit formulae for most order-1 invariants were given in [Nowik $\geq 2006$ ]; the same article and [Nowik 2001a] gave explicit formulae for the values of the remaining order-1 invariants on all embeddings. Earlier work on existence and explicit formulae for small subclasses of invariants includes [Max and Banchoff 1981; Goryunov 1997; Nowik 2000; 2001b]. Here we classify all finite-order invariants of order $n>1$, and show that they are all functions of the universal order-1 invariant constructed in [Nowik 2004].

The structure of the paper is as follows. In Section 2 we summarize the necessary background, defining finite-order invariants of immersions of a closed orientable surface into $\mathbb{R}^{3}$. Given a surface $F$, a regular homotopy class $\mathscr{A}$ of immersions of $F$ into $\mathbb{R}^{3}$, and an abelian group $\mathbb{G}$, we define $V_{n}$ to be the group of all invariants on $\mathscr{A}$ of order at most $n$ with values in $\mathbb{G}$. We present a group $\Delta_{n}=\Delta_{n}(\mathbb{G})$ and an injection $\mu_{n}: V_{n} / V_{n-1} \rightarrow \Delta_{n}$. The question of classifying all finite-order invariants then becomes the question of finding the image of $\mu_{n}$. In Section 3 we state our classification. We specify a subgroup $E_{n} \subseteq \Delta_{n}$ which we claim to be the image of $\mu_{n}$. In Section 4 we show that $\mu_{n}\left(V_{n}\right) \supseteq E_{n}$ by explicit construction. In Section 5 we show that $\mu_{n}\left(V_{n}\right) \subseteq E_{n}$.

MSC2000: 57M99, 57R42.

Keywords: immersions of surfaces, finite order invariants.

Partially supported by the Minerva Foundation. 


\section{Background}

For additional details on this section's material, see [Nowik 2004]. Given a closed oriented surface $F$, let $\operatorname{Imm}\left(F, \mathbb{R}^{3}\right)$ denote the space of all immersions of $F$ into $\mathbb{R}^{3}$, with the $C^{1}$ topology. A CE point of an immersion $i: F \rightarrow \mathbb{R}^{3}$ is a point of self-intersection of $i$ for which the local stratum in $\operatorname{Imm}\left(F, \mathbb{R}^{3}\right)$ corresponding to the self-intersection has codimension one. We distinguish four basic types of CEs named $E, H, T, Q$. (See Figure 1.) The four basic CE types are then further divided into twelve types, according to the orientations of the various sheets involved, which we name $E^{0}, E^{1}, E^{2}, H^{1}, H^{2}, T^{0}, T^{1}, T^{2}, T^{3}, Q^{2}, Q^{3}, Q^{4}$.

A coörientation for a $\mathrm{CE}$ is a choice of one of the two sides of the local stratum corresponding to the CE. All but two of the above CE types are nonsymmetric in the sense that the two sides of the local stratum may be distinguished via the local configuration of the $\mathrm{CE}$, and for those ten $\mathrm{CE}$ types, permanent coorientations for the corresponding strata are chosen once and for all. The two exceptions are $H^{1}$ and $Q^{2}$ which are completely symmetric.

We fix a closed oriented surface $F$ and a regular homotopy class $\mathscr{A}$ of immersions of $F$ into $\mathbb{R}^{3}$ (that is, $\mathscr{A}$ is a connected component of $\operatorname{Imm}\left(F, \mathbb{R}^{3}\right)$ ). We denote by $I_{n} \subseteq \mathscr{A}(n \geq 0)$ the space of all immersions in $\mathscr{A}$ which have precisely $n$ CE points (the self-intersection being stable elsewhere). In particular, $I_{0}$ is the space of all stable immersions in $\mathscr{A}$. For an immersion $i: F \rightarrow \mathbb{R}^{3}$ having a CE
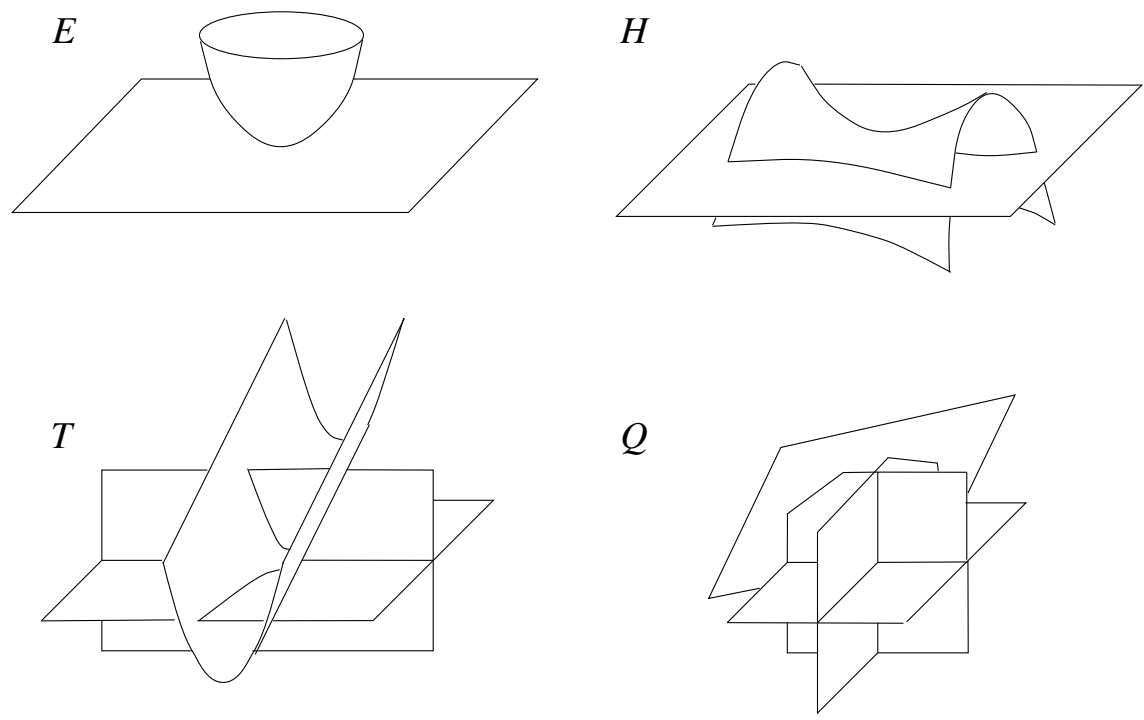

Figure 1. A slight lowering of the topmost sheet in each diagram will produce the corresponding $\mathrm{CE}$. 
located at $p \in \mathbb{R}^{3}$, the degree $d_{p}(i) \in \mathbb{Z}$ of $i$ at $p$ is defined as follows: Push each sheet of $F$ that passes through $p$ a bit into its preferred side determined by the orientation of $F$, obtaining a new immersion $\hat{\imath}$ that misses $p$, and we define $d_{p}(i)$ to be the degree of the map $\hat{\imath}: F \rightarrow \mathbb{R}^{3}-\{p\}$. We then define $C_{p}(i)$ to be the expression $R_{m}^{a}$ where $R^{a}$ is the symbol describing the configuration of the CE of $i$ at $p$ (one of the twelve symbols above) and $m=d_{p}(i) . \mathscr{C}_{n}$ denotes the set of all unordered $n$-tuples of expressions $R_{m}^{a}$ with $R^{a}$ one of our twelve symbols and $m \in \mathbb{Z}$. A map $C: I_{n} \rightarrow \mathscr{C}_{n}$ is defined by $C(i)=\left[C_{p_{1}}(i), \ldots, C_{p_{n}}(i)\right] \in \mathscr{C}_{n}$ where $p_{1}, \ldots, p_{n}$ are the $n$ CE points of $i$. The map $C: I_{n} \rightarrow \mathscr{C}_{n}$ is surjective.

Given an immersion $i \in I_{n}$, a temporary coorientation for $i$ is a choice of coorientation at each of the $n$ CE points $p_{1}, \ldots, p_{n}$ of $i$. Given a temporary coorientation $\mathfrak{T}$ for $i$ and a subset $A \subseteq\left\{p_{1}, \ldots, p_{n}\right\}$, we define $i_{\mathfrak{T}, A} \in I_{0}$ to be the immersion obtained from $i$ by resolving all CEs of $i$ at points of $A$ into the positive side with respect to $\mathfrak{T}$, and all CEs not in $A$ into the negative side. Now let $\mathbb{G}$ be any abelian group and let $f: I_{0} \rightarrow \mathbb{G}$ be an invariant, that is, a function constant on each connected component of $I_{0}$. Given $i \in I_{n}$ and a temporary coorientation $\mathfrak{T}$ for $i$, $f^{\mathfrak{T}}(i)$ is defined as follows:

$$
f^{\mathfrak{T}}(i)=\sum_{A \subseteq\left\{p_{1}, \ldots, p_{n}\right\}}(-1)^{n-|A|} f\left(i_{\mathfrak{T}, A}\right)
$$

where $|A|$ is the number of elements in $A$. If $\mathfrak{T}, \mathfrak{T}^{\prime}$ are temporary coorientations for the same immersion $i$ then $f^{\mathfrak{T}}(i)= \pm f^{\mathfrak{T}^{\prime}}(i)$, so the condition $f^{\mathfrak{T}}(i)=0$ is independent of the temporary coorientation $\mathfrak{T}$. An invariant $f: I_{0} \rightarrow \mathbb{G}$ is called of finite order if there is $n$ such that $f^{\mathfrak{T}}(i)=0$ for all $i \in I_{n+1}$. The minimal such $n$ is called the order of $f$. The group of all invariants on $I_{0}$ of order at most $n$ is denoted $V_{n}$.

Let $f \in V_{n}$. If $i \in I_{n}$ has at least one CE of type $H^{1}$ or $Q^{2}$ and $\mathfrak{T}$ is a temporary coorientation for $i$, then $2 f^{\mathfrak{T}}(i)=0$, by [Nowik 2004, Proposition 3.5], and so in this case $f^{\mathfrak{T}}(i)$ is independent of $\mathfrak{T}$. Using this fact, $f \in V_{n}$ will induce a function $\hat{f}: I_{n} \rightarrow \mathbb{G}$ as follows: For any $i \in I_{n}$ we set $\hat{f}(i)=f^{\mathfrak{T}}(i)$, where if $i$ includes at least one CE of type $H^{1}$ or $Q^{2}$ then $\mathfrak{T}$ is arbitrary, and if all CEs of $i$ are not of type $H^{1}$ or $Q^{2}$ then the permanent coorientation is used for all CEs of $i$. (If $f \in V_{n}$ then we are not inducing such function on $I_{k}$ for $0<k<n$ ). In order not to need to distinguish between the above two cases, we will define for any $i \in I_{n}$, a proper coorientation to be a choice of coorientation for each of the CEs of $i$, which is the permanent coorientation for each CE which is not of type $H^{1}$ or $Q^{2}$. In these terms we may simply say that for any $i \in I_{n}, \hat{f}(i)$ is defined as $f^{\mathfrak{T}}(i)$ with $\mathfrak{T}$ a proper coorientation for $i$. For $f \in V_{n}$ and $i, j \in I_{n}$, if $C(i)=C(j)$ then $\hat{f}(i)=\hat{f}(j)$ [Nowik 2004, Proposition 3.8], so any $f \in V_{n}$ induces a well defined function $\mu_{n}(f): \mathscr{C}_{n} \rightarrow \mathbb{G}$. The map $f \mapsto \mu_{n}(f)$ induces an injection $\mu_{n}: V_{n} / V_{n-1} \rightarrow \mathscr{C}_{n}^{*}$ 
where $\mathscr{C}_{n}^{*}$ is the group of all functions from $\mathscr{C}_{n}$ to $\mathbb{G}$. Finding the image of $\mu_{n}$ for all $n$ gives a classification of all finite order invariants, which is what we do in this work (Theorem 3.2). For order-1 invariants this has been done in [Nowik 2004].

The proof that if $C(i)=C(j)$ then $\hat{f}(i)=\hat{f}(j)$ uses the notion of AB equivalence which we now recall. A regular homotopy between two immersions in $I_{n}$ is called an $\mathrm{AB}$ equivalence if it is alternatingly of type $\mathrm{A}$ and $\mathrm{B}$, where

(1) $J_{t}: F \rightarrow \mathbb{R}^{3}(0 \leq t \leq 1)$ is of type $\mathrm{A}$ if it is of the form $J_{t}=U_{t} \circ i \circ V_{t}$ where $i: F \rightarrow \mathbb{R}^{3}$ is an immersion and $U_{t}: \mathbb{R}^{3} \rightarrow \mathbb{R}^{3}, V_{t}: F \rightarrow F$ are isotopies.

(2) $J_{t}: F \rightarrow \mathbb{R}^{3}(0 \leq t \leq 1)$ is of type B if $J_{0} \in I_{n}$ and there are little balls $B_{1}, \ldots, B_{n} \subseteq \mathbb{R}^{3}$ centered at the $n \mathrm{CE}$ points of $J_{0}$ such that $J_{t}$ fixes $U=$ $\left(J_{0}\right)^{-1}\left(\bigcup_{k} B_{k}\right)$ and moves $F-U$ within $\mathbb{R}^{3}-\bigcup_{k} B_{k}$.

The subgroup $\Delta_{n}=\Delta_{n}(\mathbb{G}) \subseteq \mathscr{C}_{n}^{*}$ which is shown in [Nowik 2004] to contain the image of $\mu_{n}$, is defined as the set of functions in $\mathscr{C}_{n}^{*}$ satisfying relations which we write as relations on the symbols in $\mathscr{C}_{1}$; e.g., $T_{m}^{0}=T_{m}^{3}$ will stand for the set of all relations of the form $g\left(\left[T_{m}^{0}, Z_{2}, \ldots, Z_{n}\right]\right)=g\left(\left[T_{m}^{3}, Z_{2}, \ldots, Z_{n}\right]\right)$ with arbitrary $Z_{2}, \ldots, Z_{n} \in \mathscr{C}_{1}$. The relations defining $\Delta_{n}$ are:

- $E_{m}^{2}=-E_{m}^{0}=H_{m}^{2}, \quad E_{m}^{1}=H_{m}^{1}$.

- $T_{m}^{0}=T_{m}^{3}, T_{m}^{1}=T_{m}^{2}$.

- $2 H_{m}^{1}=0, \quad H_{m}^{1}=H_{m-1}^{1}$.

- $2 Q_{m}^{2}=0, Q_{m}^{2}=Q_{m-1}^{2}$.

- $H_{m}^{2}-H_{m-1}^{2}=T_{m}^{3}-T_{m}^{2}$.

- $Q_{m}^{4}-Q_{m}^{3}=T_{m}^{3}-T_{m-1}^{3}, Q_{m}^{3}-Q_{m}^{2}=T_{m}^{2}-T_{m-1}^{2}$.

The above relations are easily solved, namely, there exists a subset $X \subseteq \mathscr{C}_{1}$ such that any $g \in \Delta_{1}$ may be defined by arbitrarily assigning values in $\mathbb{G}$ to each $Z \in X$, and assigning values in $\mathbb{B}=\{x \in \mathbb{G}: 2 x=0\}$ to the two symbols $H_{0}^{1}, Q_{0}^{2}$. Once this is done, the value of $g$ on all other symbols is uniquely defined, as combinations, with integer coefficients, of the values on the symbols in $Y=X \cup\left\{H_{0}^{1}, Q_{0}^{2}\right\}$. In [Nowik 2004] the set $X$ is chosen as $\left\{T_{m}^{a}\right\}_{a=2,3, m \in \mathbb{Z}} \cup\left\{H_{0}^{2}\right\}$. In [Nowik $\geq 2006$ ] there is an improved choice $X=\left\{T_{m}^{2}\right\}_{m \in \mathbb{Z}} \cup\left\{H_{m}^{2}\right\}_{m \in \mathbb{Z}}$, for which the mentioned integer coefficients attain simpler form. We define the group $L_{1}$ by the abelian group presentation

$$
L_{1}=\left\langle\left\{a_{Z}\right\}_{Z \in X} \cup\{b, c\} \mid 2 b=2 c=0\right\rangle .
$$

(This is the group $\mathbb{G}_{U}$ appearing in [Nowik 2004], with generators relabeled.) Let $g_{1}^{U} \in \Delta_{1}\left(L_{1}\right)$ be the function in $\Delta_{1}\left(L_{1}\right)$ uniquely defined by $g_{1}^{U}(Z)=a_{Z}$ for $Z \in X$, $g_{1}^{U}\left(H_{0}^{1}\right)=b, g_{1}^{U}\left(Q_{0}^{2}\right)=c$. The main result of [Nowik 2004] is that for any closed orientable surface $F$, regular homotopy class $\mathscr{A}$ of immersions of $F$ into $\mathbb{R}^{3}$ and 
abelian group $\mathbb{G}$, the injection $\mu_{1}: V_{1} / V_{0} \rightarrow \Delta_{1}$ is surjective. This is shown by proving the existence of an order-1 invariant $f_{1}^{U}: I_{0} \rightarrow L_{1}$ satisfying $\mu_{1}\left(f_{1}^{U}\right)=g_{1}^{U}$.

\section{Statement of classification}

Let $X \subseteq Y \subseteq \mathscr{C}_{1}$ be as above. If we denote the elements of $Y$ by $y_{i}$ and those of $\mathscr{C}_{1}$ by $c_{j}$ (note $\left\{y_{i}\right\} \subseteq\left\{c_{j}\right\}$ ), then as mentioned above there are integers $n_{j}^{i}$ (where for each $j, n_{j}^{i} \neq 0$ for only finitely many values of $i$ ) such that for any $\mathbb{G}$, any $g \in \Delta_{1}(\mathbb{G})$, and any $j, g\left(c_{j}\right)=\sum_{i} n_{j}^{i} g\left(y_{i}\right)$. Since the same relations, applied to each term of an $n$-tuple separately, define $\Delta_{n}$, it follows that a function $g \in \Delta_{n}(\mathbb{G})$ may be assigned arbitrary values in $\mathbb{G}$ for any unordered $n$-tuple of elements of $X$ and arbitrary values in $\mathbb{B}$ for all $n$-tuples of elements of $Y$ which include $H_{0}^{1}$ or $Q_{0}^{2}$ at least once. Once this is done, the value of $g$ on all other $n$-tuples in $\mathscr{C}_{n}$ is uniquely determined by:

$$
g\left(\left[c_{j_{1}}, \ldots, c_{j_{n}}\right]\right)=\sum_{i_{1}, \ldots, i_{n}} n_{j_{1}}^{i_{1}} n_{j_{2}}^{i_{2}} \cdots n_{j_{n}}^{i_{n}} g\left(\left[y_{i_{1}}, \ldots, y_{i_{n}}\right]\right) .
$$

Indeed the fact that we are dealing with unordered $n$-tuples poses no problem. For the sake of the above calculation one may think of $g$ as a symmetric function of ordered $n$-tuples.

We will now define $E_{n} \subseteq \Delta_{n}$ by two additional restrictions on the functions $g \in \Delta_{n}$. Thanks to the discussion of the previous paragraph, we may state the additional restrictions in terms of the values of $g$ on $n$-tuples of elements of $Y$ only. Given an unordered $n$-tuple $z$ of elements of $Y$, we define $m_{H_{0}^{1}}(z)$ and $m_{Q_{0}^{2}}(z)$ as the number of times that $H_{0}^{1}$ and $Q_{0}^{2}$ appear in $z$ respectively. We define $r(z)$, the repetition of $H_{0}^{1}$ and $Q_{0}^{2}$ in $z$, as

$$
r(z)=\max \left(0, m_{H_{0}^{1}}(z)-1\right)+\max \left(0, m_{Q_{0}^{2}}(z)-1\right) .
$$

Definition 3.1. Given an abelian group $\mathbb{G}, E_{n}=E_{n}(\mathbb{G}) \subseteq \Delta_{n}(\mathbb{G})$ is the subgroup consisting of all functions $g \in \Delta_{n}(\mathbb{G})$ that satisfy the following two additional restrictions:

(1) When $n \geq 3, g$ must satisfy the relation $H_{0}^{1} H_{0}^{1} Q_{0}^{2}=H_{0}^{1} Q_{0}^{2} Q_{0}^{2}$. By this we mean that $g\left(\left[H_{0}^{1}, H_{0}^{1}, Q_{0}^{2}, Z_{4}, \ldots, Z_{n}\right]\right)=g\left(\left[H_{0}^{1}, Q_{0}^{2}, Q_{0}^{2}, Z_{4}, \ldots, Z_{n}\right]\right)$ for any $Z_{4}, \ldots, Z_{n} \in Y$.

(2) For any unordered $n$-tuple $z$ of elements of $Y, g(z) \in 2^{r(z)} \mathbb{G}$; that is, there exists an element $a \in \mathbb{G}$ such that $g(z)=2^{r(z)} a$. (Whenever $r(z)>0$, in particular $H_{0}^{1}$ or $Q_{0}^{2}$ does appear in $z$, so in fact we have $g(z) \in \mathbb{B} \cap 2^{r(z)} \mathbb{G}$.)

In this work we prove: 
Theorem 3.2. For any closed orientable surface $F$, regular homotopy class $\mathscr{A}$ of immersions of $F$ into $\mathbb{R}^{3}$ and abelian group $\mathbb{G}$, the image of the injection $\mu_{n}$ : $V_{n} / V_{n-1} \rightarrow \Delta_{n}$ is $E_{n}$.

Furthermore, for any $f \in V_{n}$, there is a function (not homomorphism) $s: L_{1} \rightarrow \mathbb{G}$ such that $f=s \circ f_{1}^{U}$.

\section{Proof that $\mu_{n}\left(V_{n}\right) \supseteq E_{n}$}

We define algebraic structures $K \subseteq L \subseteq M$, where $L$ is a commutative ring, $K$ is a subring of $L$, and $M$ is a module over $K$. We define $L$ as the ring of formal power series with integer coefficients, with variables $\left\{a_{i}\right\}_{i \in X} \cup\{b, c\}$ and with the following relations:

- $2 b=2 c=0$.

- $b^{2} c=b c^{2}$.

We emphasize that though there is an infinite set of variables, any given power series may include only finitely many monomials of any given degree $n$. Given a monomial $p$, we define $m_{b}(p)$ as the multiplicity of $b$ in $p$, and likewise for $c$. We define $r(p)$, (the repetition of $b$ and $c$ in $p$ ), as

$$
r(p)=\max \left(0, m_{b}(p)-1\right)+\max \left(0, m_{c}(p)-1\right) .
$$

Then $r(p)$ is preserved under the relations in $L$ and so is well defined on equivalence classes of monomials. Now $K \subseteq L$ is defined to be the subring of power series including only the variables $\left\{a_{i}\right\}_{i \in X}$. On the other hand we extend $L$ to a larger structure $M$ which will be a module over $K$, as follows: For each monomial $p \in L$ with coefficient 1 (more precisely, an equivalence class of such monomials), we adjoin a new element $\zeta_{p}$ satisfying the relation $2^{r(p)} \zeta_{p}=p$. The new elements $\zeta_{p}$ will be considered monomials of the same degree as the corresponding $p$, and will appear as terms in our formal power series. (Indeed, one can think of $\zeta_{p}$ as $2^{-r(p)} p$.) Note that if $r(p)=0$ then $\zeta_{p}=p$, in particular, $\zeta_{1}=1$ and $\zeta_{e}=e$ for each generating variable $e$. We note that the ring structure of $L$ cannot be extended to $M$, as we would get contradictions such as $b^{3}=b \cdot b^{2}=b \cdot 2 \cdot \zeta_{b^{2}}=0$ (since $r\left(b^{2}\right)=1$ and $2 b=0$ ). We do however extend the action of $K$ on $L$ (as a subring) to an action of $K$ on $M$, turning $M$ into a module over $K$, as follows: If $k \in K, p \in L$ are monomials then $k \cdot \zeta_{p}=\zeta_{k p}$. This is extended in the natural way to an action of power series in $K$ on power series in $M$. For each $n \geq 0$ we denote by $K_{n} \subseteq L_{n} \subseteq M_{n}$ the additive subgroups of $K \subseteq L \subseteq M$ respectively, generated by the monomials of degree $n$. (Recall that $\zeta_{p} \in M$ is considered a monomial of the same degree as $p$.) We note that $L_{1}$ coincides with our previous definition and that $L_{1}=M_{1}$. We have $L_{1}=K_{1} \oplus S$ where $S \subseteq L_{1}$ is the four element subgroup generated by $b, c$. 
We now obtain a function $\mathscr{F}: L_{1} \rightarrow M$ as follows. We first define $\mathscr{F}: K_{1} \rightarrow K$ as the group homomorphism from the additive group $K_{1}$ to the multiplicative group of invertible elements in $K$, which is given on generators by

$$
\mathscr{F}\left(a_{i}\right)=\sum_{n=0}^{\infty} a_{i}^{n} .
$$

These are indeed invertible elements, giving

$$
\mathscr{F}\left(-a_{i}\right)=\left(\sum_{n=0}^{\infty} a_{i}^{n}\right)^{-1}=1-a_{i} .
$$

We then define $\mathscr{F}: S \rightarrow M$ explicitly on the four elements of $S$ :

(1) $\mathscr{F}(0)=1$.

(2) $\mathscr{F}(b)=\sum_{n=0}^{\infty} \zeta b^{n}$.

(3) $\mathscr{F}(c)=\sum_{n=0}^{\infty} \zeta_{c^{n}}$.

(4) $\mathscr{F}(b+c)=1+b+c+\sum_{n=2}^{\infty}\left(\zeta_{b^{n}}+\zeta_{c^{n}}+\zeta_{b c^{n-1}}\right)$.

Finally, $\mathscr{F}: L_{1} \rightarrow M$ is defined as follows: Any element in $L_{1}$ is uniquely written as $k+s$ with $k \in K_{1}, s \in S$, and we define $\mathscr{F}(k+s)=\mathscr{F}(k) \mathscr{F}(s)$ where the product on the right is the action of $K$ on $M$.

Lemma 4.1. For any $k \in K_{1}, l \in L_{1}$ we have $\mathscr{F}(k+l)=\mathscr{F}(k) \mathscr{F}(l)$.

Proof. Let $l=k^{\prime}+s$. Then

$$
\mathscr{F}(k+l)=\mathscr{F}\left(k+k^{\prime}+s\right)=\mathscr{F}\left(k+k^{\prime}\right) \mathscr{F}(s)=\mathscr{F}(k) \mathscr{F}\left(k^{\prime}\right) \mathscr{F}(s)=\mathscr{F}(k) \mathscr{F}(l) .
$$

Lemma 4.2. For any $l \in L_{1}$ we have $\mathscr{F}(l)=1+l+T_{2}$, where $T_{2}$ stands for the higher order terms of the given series, that is, some power series in $M$ which includes only monomials of degree at least 2.

Proof. For $l= \pm a_{i}$ and for each $l \in S$, this follows by direct inspection of the formulae above. It then follows for all $l \in L_{1}$.

Definition 4.3. For any $(n+1)$-tuple $\left(l ; l_{1}, l_{2}, \ldots, l_{n}\right)$ of elements of $L_{1}$, define

$$
\mathscr{F}^{\prime}\left(l ; l_{1}, \ldots, l_{n}\right)=\sum_{A \subseteq\{1, \ldots, n\}}(-1)^{n-|A|} \mathscr{F}_{\mathcal{F}}\left(l+\sum_{i \in A} l_{i}\right) .
$$

We will repeatedly use the following splitting of the sum defining $\mathscr{F}^{\prime}$ :

$$
\mathscr{F}^{\prime}\left(l ; l_{1}, \ldots, l_{n}\right)=\sum_{A \subseteq\{2, \ldots, n\}}(-1)^{n-|A|-1}\left(\mathscr{F}\left(l+l_{1}+\sum_{i \in A} l_{i}\right)-\mathscr{F}\left(l+\sum_{i \in A} l_{i}\right)\right)
$$


Proposition 4.4. For any $(n+1)$-tuple $\left(l ; l_{1}, \ldots, l_{n}\right)$ of elements of $L_{1}$, we have

$$
\mathscr{F}^{\prime}\left(l ; l_{1}, \ldots, l_{n}\right)=l_{1} l_{2} \ldots l_{n}+T_{n+1},
$$

where $l_{1} l_{2} \cdots l_{n}$ is the product in $L$, thought of as an element in $M$, and $T_{n+1}$ stands for higher order terms, that is, some power series in $M$ including only monomials of degree at least $n+1$.

Proof. From the splitting presented above of the sum defining $\mathscr{F}^{\prime}$ we see that $\mathscr{F}^{\prime}\left(l ; 0, l_{2}, \ldots, l_{n}\right)=0$, in which case the statement holds, and similarly if any $l_{i}=0$. So we assume from now on that all $l_{i}$ are nonzero, and we proceed by induction on the sum of lengths of $l_{1}, \ldots, l_{n}$ in terms of the generators of $L_{1}$.

For total length 0 we have $\mathscr{F}^{\prime}(l ; \varnothing)=\mathscr{F}(l)=1+l+T_{2}=1+T_{1}$ by Lemma 4.2. Defining an empty product to be 1 , the statement holds in this case.

For total length greater than 0 , if say $l_{1}$ is not a generator and $l_{1}=l_{1}^{\prime}+l_{1}^{\prime \prime}$ where $l_{1}^{\prime}$ and $l_{1}^{\prime \prime}$ have shorter length than $l_{1}$ in terms of the generators, then $\mathscr{F}^{\prime}\left(l ; l_{1}, \ldots, l_{n}\right)$

$$
\begin{aligned}
& =\sum_{A \subseteq\{2, \ldots, n\}}(-1)^{n-|A|-1}\left(\mathscr{F}\left(l+l_{1}+\sum_{i \in A} l_{i}\right)-\mathscr{F}\left(l+\sum_{i \in A} l_{i}\right)\right) \\
& =\sum_{A \subseteq\{2, \ldots, n\}}(-1)^{n-|A|-1}\left(\mathscr{F}\left(l+l_{1}^{\prime}+l_{1}^{\prime \prime}+\sum_{i \in A} l_{i}\right)-\mathscr{F}\left(l+l_{1}^{\prime}+\sum_{i \in A} l_{i}\right)\right) \\
& \quad+\sum_{A \subseteq\{2, \ldots, n\}}(-1)^{n-|A|-1}\left(\mathscr{F}\left(l+l_{1}^{\prime}+\sum_{i \in A} l_{i}\right)-\mathscr{F}\left(l+\sum_{i \in A} l_{i}\right)\right) \\
& =\mathscr{F}^{\prime}\left(l+l_{1}^{\prime} ; l_{1}^{\prime \prime}, l_{2}, \ldots, l_{n}\right)+\mathscr{F}^{\prime}\left(l ; l_{1}^{\prime}, l_{2}, \ldots, l_{n}\right) \\
& =l_{1}^{\prime \prime} l_{2} \cdots l_{n}+T_{n+1}+l_{1}^{\prime} l_{2} \cdots l_{n}+T_{n+1}=l_{1} l_{2} \cdots l_{n}+T_{n+1} .
\end{aligned}
$$

We are thus left with the case where all the $l_{i}$ are generators or minus generators of $L_{1}$; that is, they are of the form $\pm e$ where $e \in\left\{a_{i}\right\}_{i \in X} \cup\{b, c\}$. If one of the $l_{i}$ is in $K_{1}$, say $l_{1}=k \in K_{1}$, then

$$
\begin{aligned}
\mathscr{F}^{\prime}\left(l ; k, l_{2}, \ldots, l_{n}\right) & =\sum_{A \subseteq\{2, \ldots, n\}}(-1)^{n-|A|-1}\left(\mathscr{F}\left(l+k+\sum_{i \in A} l_{i}\right)-\mathscr{F}\left(l+\sum_{i \in A} l_{i}\right)\right) \\
& =(\mathscr{F}(k)-1) \sum_{A \subseteq\{2, \ldots, n\}}(-1)^{n-1-|A|} \mathscr{F}\left(l+\sum_{i \in A} l_{i}\right) \\
& =(\mathscr{F}(k)-1) \mathscr{F}^{\prime}\left(l ; l_{2}, \ldots, l_{n}\right) \\
& =\left(k+T_{2}\right)\left(l_{2} \cdots l_{n}+T_{n}\right)=k l_{2} \cdots l_{n}+T_{n+1} .
\end{aligned}
$$


The second equality follows from Lemma 4.1. The first term in the fourth equality follows from Lemma 4.2 (and the $T_{2}$ is in $K$ ). If $n=1$ the product $l_{2} \cdots l_{n}$ is an empty product, equaling 1 , as defined above.

So assume now that all $l_{1}, \ldots, l_{n}$ are $b$ and $c$. Assume $k$ of them are $b$ and $n-k$ of them are $c$. We first deal with the case $k=n$, so that $l_{1}, \ldots, l_{n}$ all equal $b$ (the case $k=0$ is identical). Since $2 b=0$ (and since there are $2^{n-1}$ odd sized and $2^{n-1}$ even sized subsets of $\{1, \ldots, n\})$, we get

$$
\mathscr{F}^{\prime}(l ; b, b, \ldots, b)=\sum_{A \subseteq\{1, \ldots, n\}}(-1)^{n-|A|} \mathscr{F}(l+|A| b)= \pm 2^{n-1}(\mathscr{F}(l+b)-\mathscr{F}(l)) .
$$

Now letting $l=k+s$ with $k \in K_{1}, s \in S$, we get:

$$
\begin{aligned}
\pm 2^{n-1}(\mathscr{F}(k+s+b)-\mathscr{F}(k+s)) & = \pm \mathscr{F}(k) 2^{n-1}(\mathscr{F}(s+b)-\mathscr{F}(s)) \\
& = \pm\left(1+T_{1}\right) 2^{n-1}(\mathscr{F}(s+b)-\mathscr{F}(s)) .
\end{aligned}
$$

Since multiplication by $1+T_{1} \in K$ leaves the lowest order term unchanged, we may assume that we have only $\pm 2^{n-1}(\mathscr{F}(s+b)-\mathscr{F}(s))$. If $s=0$ or $b$ then

$$
\pm 2^{n-1}(\mathscr{F}(s+b)-\mathscr{F}(s))= \pm 2^{n-1} \sum_{m=1}^{\infty} \zeta_{b^{m}}=b^{n}+T_{n+1}
$$

since $r\left(b^{m}\right)=m-1$ and so $2^{n-1} \zeta_{b^{m}}=0$ for $m<n$ and $2^{n-1} \zeta_{b^{n}}=b^{n}$. (The \pm was dropped since $2 b^{n}=0$.) If $s=c$ or $c+b$ then we get

$$
\pm 2^{n-1}(\mathscr{F}(s+b)-\mathscr{F}(s))= \pm 2^{n-1}\left(b+\sum_{m=2}^{\infty}\left(\zeta_{b^{m}}+\zeta_{b c^{m-1}}\right)\right)=b^{n}+T_{n+1}
$$

since again $r\left(b^{m}\right)=m-1$, but furthermore $r\left(b c^{m-1}\right)=m-2$ and so $2^{n-1} \zeta_{b c^{m-1}}=0$ for $m \leq n$.

We are left with the case of $b$ appearing $k$ times and $c$ appearing $n-k$ times, with $0<k<n$. Since $2 b=2 c=0$, we get

$$
\begin{aligned}
\mathscr{F}^{\prime}(l ; b, \ldots, b, c, \ldots, c) & =\sum_{\substack{B \subseteq\{1, \ldots, k\} \\
C \subseteq\{k+1, \ldots, n\}}}(-1)^{n-|B|-|C|} \mathscr{F}(l+|B| b+|C| c) \\
& = \pm 2^{n-2}(\mathscr{F}(l+b+c)-\mathscr{F}(l+b)-\mathscr{F}(l+c)+\mathscr{F}(l)) .
\end{aligned}
$$

As before, by writing $l=k+s$ and factoring out $\mathscr{F}(k)$, we may assume $l=s \in S$. For each of the four elements $s \in S$ we get

$$
\begin{aligned}
\pm 2^{n-2}(\mathscr{F}(s+b+c)-\mathscr{F}(s+b)-\mathscr{F}(s+c)+\mathscr{F}(s)) & = \pm 2^{n-2} \sum_{m=2}^{\infty} \zeta_{b c^{m-1}} \\
& =b c^{n-1}+T_{n+1}=b^{k} c^{n-k}+T_{n+1},
\end{aligned}
$$


since $r\left(b c^{m-1}\right)=m-2$ and so $2^{n-2} \zeta_{b c^{m-1}}=0$ for $m<n$, and $2^{n-2} \zeta_{b c^{n-1}}=b c^{n-1}$.

Extending the definition of $g_{1}^{U}$ from Section 2, let $g_{n}^{U}$ be the unique function in $\Delta_{n}\left(M_{n}\right)$ defined on unordered $n$-tuples $y_{i_{1}}, \ldots, y_{i_{n}}$ of elements of $Y$ by

$$
g_{n}^{U}\left(\left[y_{i_{1}}, \cdots, y_{i_{n}}\right]\right)=g_{1}^{U}\left(y_{i_{1}}\right) g_{1}^{U}\left(y_{i_{2}}\right) \cdots g_{1}^{U}\left(y_{i_{n}}\right) \in L_{n} \subseteq M_{n}
$$

(the product being that in $L$ ). By the construction of $M_{n}$, in fact, $g_{n}^{U}$ lies in $E_{n}\left(M_{n}\right)$. The equality with which we have defined $g_{n}^{U}$ on $n$-tuples of elements of $Y$ is true for all unordered $n$-tuples $\left[c_{i_{1}}, \ldots, c_{i_{n}}\right] \in \mathscr{C}_{n}$. Indeed,

$$
\begin{aligned}
g_{n}^{U}\left(\left[c_{j_{1}}, \ldots, c_{j_{n}}\right]\right) & =\sum_{i_{1}, \ldots, i_{n}} n_{j_{1}}^{i_{1}} \cdots n_{j_{n}}^{i_{n}} g_{n}^{U}\left(\left[y_{i_{1}}, \ldots, y_{i_{n}}\right]\right) \\
& =\sum_{i_{1}, \ldots, i_{n}} n_{j_{1}}^{i_{1}} \cdots n_{j_{n}}^{i_{n}} g_{1}^{U}\left(y_{i_{1}}\right) \cdots g_{1}^{U}\left(y_{i_{n}}\right) \\
& =\left(\sum_{i_{1}} n_{j_{1}}^{i_{1}} g_{1}^{U}\left(y_{i_{1}}\right)\right) \cdots\left(\sum_{i_{n}} n_{j_{n}}^{i_{n}} g_{1}^{U}\left(y_{i_{n}}\right)\right) \\
& =g_{1}^{U}\left(c_{j_{1}}\right) \cdots g_{1}^{U}\left(c_{j_{n}}\right) .
\end{aligned}
$$

We now look at the invariant $\mathscr{F}_{F} \circ f_{1}^{U}: I_{0} \rightarrow M$. Take $i \in I_{n}$, with CEs at $\left\{p_{1}, \ldots, p_{n}\right\}$, and let $\mathfrak{T}$ be a proper coorientation for $i$. By Proposition 4.4 and since $\mu_{1}\left(f_{1}^{U}\right)=g_{1}^{U}$,

$$
\begin{aligned}
\left(\mathscr{F}_{F} \circ f_{1}^{U}\right)^{\mathfrak{T}}(i) & =\sum_{A \subseteq\left\{p_{1}, \ldots, p_{n}\right\}}(-1)^{n-|A|} \mathscr{F}_{\mathscr{F}} \circ f_{1}^{U}\left(i_{\mathfrak{T}, A}\right) \\
& =\sum_{A \subseteq\left\{p_{1}, \ldots, p_{n}\right\}}(-1)^{n-|A|}\left(\mathscr{F}\left(f_{1}^{U}\left(i_{\mathfrak{T}, \varnothing}\right)+\sum_{p_{j} \in A} g_{1}^{U}\left(C_{p_{j}}(i)\right)\right)\right) \\
& =\mathscr{F}^{\prime}\left(f_{1}^{U}\left(i i_{\mathfrak{T}, \varnothing}\right) ; g_{1}^{U}\left(C_{p_{1}}(i)\right), \ldots, g_{1}^{U}\left(C_{p_{n}}(i)\right)\right) \\
& =g_{1}^{U}\left(C_{p_{1}}(i)\right) g_{1}^{U}\left(C_{p_{2}}(i)\right) \cdots g_{1}^{U}\left(C_{p_{n}}(i)\right)+T_{n+1} \\
& =g_{n}^{U}(C(i))+T_{n+1} .
\end{aligned}
$$

Now let $\pi_{n}: M \rightarrow M_{n}$ be the projection, and define $f_{n}^{U}: I_{0} \rightarrow M_{n}$ to be the invariant given by $f_{n}^{U}=\pi_{n} \circ \mathscr{F}_{F} \circ f_{1}^{U}$. Then by the formula we obtained for (F्F $\circ$ $\left.f_{1}^{U}\right)^{\mathfrak{T}}(i)$ we get that for any $i \in I_{n+1}$, and proper coorientation $\mathfrak{T}$ for $i,\left(f_{n}^{U}\right)^{\mathfrak{T}}(i)=$ $\pi_{n}\left(\left(\mathscr{F}_{\circ} \circ f_{1}^{U}\right)^{\mathfrak{T}}(i)\right)=\pi_{n}\left(g_{n+1}^{U}(C(i))+T_{n+2}\right)=0$, and for any $i \in I_{n}$ and proper $\mathfrak{T}$ for $i,\left(f_{n}^{U}\right)^{\mathfrak{T}}(i)=g_{n}^{U}(C(i))$. That is, $f_{n}^{U}$ is an invariant of order $n$ with $\mu_{n}\left(f_{n}^{U}\right)=g_{n}^{U}$. Now for an arbitrary abelian group $\mathbb{G}$, if $g \in E_{n}(\mathbb{G})$ there exists $\varphi \in \operatorname{Hom}\left(M_{n}, \mathbb{G}\right)$ such that $g=\varphi \circ g_{n}^{U}$. Then $\varphi \circ f_{n}^{U}$ is an invariant of order $n$ with $\mu_{n}\left(\varphi \circ f_{n}^{U}\right)=$ $g$. This proves that $\mu_{n}\left(V_{n}\right) \supseteq E_{n}$ for any $\mathbb{G}$. In the next section we show that $\mu_{n}\left(V_{n}\right) \subseteq E_{n}$. 


\section{Proof that $\mu_{n}\left(V_{n}\right) \subseteq E_{n}$}

For $x, y, n \geq 0$ we define $I_{n}^{x, y}$ to be the space of immersions in $I_{x+y+n}$ with $x$ designated CEs of type $H_{0}^{1}$ with choice of ordering on them, $y$ designated CEs of type $Q_{0}^{2}$ with choice of ordering on them, and a choice of coorientation for these $x+y$ CEs. The remaining $n$ CEs may be of any type and they are neither ordered nor cooriented. So the same underlying immersion appears $2^{x+y} x ! y$ ! times in $I_{n}^{x, y}$ with different choices of ordering and coorientations. Also note that $I_{n}^{0,0}=I_{n}$.

We define an $(x, y)$-invariant to be a function $f: I_{0}^{x, y} \rightarrow \mathbb{G}$ which is constant on the connected components of $I_{0}^{x, y}$. Now let $i \in I_{n}^{x, y}$ and assume the $n$ nondesignated CEs of $i$ are at $p_{1}, \ldots, p_{n}$. Given a temporary coorientation $\mathfrak{T}$ for $p_{1}, \ldots, p_{n}$ and a subset $A \subseteq\left\{p_{1}, \ldots, p_{n}\right\}$, we define $i_{\mathfrak{T}, A} \in I_{0}^{x, y}$ as before, resolving only the nondesignated CEs and keeping the order and coorientation of the designated CEs. We may then define $f^{\mathfrak{T}}(i)$ and invariants of order $n$ as before, and define $V_{n}^{x, y}$ to be the group of all $(x, y)$-invariants of order at most $n$. By the same rule as before, each $f \in V_{n}^{x, y}$ will induce a function $\hat{f}$ on $I_{n}^{x, y}$, using a proper coorientation for the nondesignated CEs. We define $C: I_{n}^{x, y} \rightarrow \mathscr{C}_{n}$ as before, using the $n$ nondesignated CEs. Again $C$ is surjective and induces an injection $\mu_{n}: V_{n}^{x, y} / V_{n-1}^{x, y} \rightarrow \Delta_{n}$. Indeed all arguments (appearing in [Nowik 2004]) showing that $\mu_{n}$ may be defined on $V_{n}$ and that $\mu_{n}\left(V_{n}\right) \subseteq \Delta_{n}$, are applicable in just the same way to show that the same is true for $V_{n}^{x, y}$. (As a first step note that, by [Nowik 2004, Proposition 3.4, proof of Proposition 3.5, Remark 3.7], for any $i, j \in I_{n}^{x, y}, C(i)=C(j)$ if and only if there is an $\mathrm{AB}$ equivalence between the underlying immersions which preserves all additional structure, i.e., brings each designated CE of $i$ to its counterpart in $j$, and with the right coorientation.) We will show that in fact $\mu_{n}\left(V_{n}^{x, y}\right) \subseteq E_{n}$ for any $x, y$. In particular we will have $\mu_{n}\left(V_{n}\right)=\mu_{n}\left(V_{n}^{0,0}\right) \subseteq E_{n}$, which is the aim of this section. The purpose of defining $V_{n}^{x, y}$ is an inductive process which will reduce $n$ but will increase $x$ and $y$.

Definition 5.1. If $i \in I_{0}^{x, y}$, we denote by $i^{\prime}$ the immersion in $I_{0}$ obtained from $i$ by resolving all $x+y$ designated CEs into the positive side determined by their chosen coorientation.

Lemma 5.2. Given $n$, assume it is known that for any $k<n$ (and any $x, y$ ), $\mu_{k}\left(V_{k}^{x, y}\right) \subseteq E_{k}$. Then for any $k<n$ and any $f \in V_{k}^{x, y}$ there exists $F \in V_{k}$ such that $f(i)=F\left(i^{\prime}\right)$ for any $i \in I_{0}^{x, y}$.

Proof. We work by induction on $k(<n)$. By assumption, $\mu_{k}(f) \in E_{k}$. From Section 4 we know that $\mu_{k}\left(V_{k}\right)$ contains $E_{k}$, so there exists $G \in V_{k}$ with $\mu_{k}(G)=$ $\mu_{k}(f)$. Let $h$ be the invariant on $I_{0}^{x, y}$ defined by $h(i)=f(i)-G\left(i^{\prime}\right)$. Then $\mu_{k}(h)=0$, so $h \in V_{k-1}^{x, y}$, so by the induction hypothesis there is $H \in V_{k-1}$ such that $h(i)=H\left(i^{\prime}\right)$ for all $i \in I_{0}^{x, y} . F=H+G$ is the required invariant on $I_{0}$. 
Before stating the next lemma we introduce some terminology. During an AB equivalence, the original CEs of the initial immersion are being dragged around. But for generic motion, there may be some finite number of times during the $\mathrm{AB}$ equivalence at each of which a single additional $\mathrm{CE}$ will be encountered, away from the original ones. We will say that the $\mathrm{AB}$ equivalence passes these additional CEs.

Lemma 5.3. Given $n$, assume it is known that for any $k<n$ (and any $x, y$ ), $\mu_{k}\left(V_{k}^{x, y}\right) \subseteq E_{k}$. Take $f \in V_{n}^{x, y}$ and let $i, j \in I_{0}^{x, y}$ be two immersions such that there is an $A B$ equivalence between them (respecting ordering and coorientations of the designated CEs), which passes precisely two additional CEs, both of which are of type $H_{0}^{1}$. Then $f(i)=f(j)$.

The same is true for $Q_{0}^{2}$.

Proof. Given $f \in V_{n}^{x, y}$ we define $f^{H} \in V_{n-1}^{x+1, y}$ and $f^{Q} \in V_{n-1}^{x, y+1}$ as follows: For $i \in I_{0}^{x+1, y}$ let $f^{H}(i)=f\left(i^{+}\right)-f\left(i^{-}\right)$where $i^{+} \in I_{0}^{x, y}$ is the immersion obtained from $i$ by resolving the $(x+1)$-th designated CE of type $H_{0}^{1}$ into the positive side determined by the chosen coorientation, and the ordering and coorientation on the remaining designated CEs remains as in $i$. Similarly $i^{-}$is defined using the negative side of the coorientation at the same CE. In the same way $f^{Q}$ is defined on $i \in I_{0}^{x, y+1}$ using the $(y+1)$-th designated CE of type $Q_{0}^{2}$. Indeed, $f^{H} \in V_{n-1}^{x+1, y}$ and $f^{Q} \in V_{n-1}^{x, y+1}$, as can be seen (for $f^{H}$, say) as follows: If $i \in I_{n}^{x+1, y}$, with CEs at $p_{1}, \ldots, p_{n}$, and $\mathfrak{T}$ is a temporary coorientation for $i$, then

$$
\begin{aligned}
\sum_{A \subseteq\left\{p_{1}, \ldots, p_{n}\right\}}(-1)^{n-|A|} f^{H}\left(i_{\mathfrak{T}, A}\right) & =\sum_{A \subseteq\left\{p_{1}, \ldots, p_{n}\right\}}(-1)^{n-|A|}\left(f\left(i_{\mathfrak{T}, A}^{+}\right)-f\left(i_{\mathfrak{T}, A}\right)\right) \\
& =\sum_{A \subseteq\left\{p_{1}, \ldots, p_{n+1}\right\}}(-1)^{n+1-|A|} f\left(\tilde{i}_{\tilde{\mathfrak{T}}, A}\right)=0,
\end{aligned}
$$

where $\tilde{i} \in I_{n+1}^{x, y}$ corresponds to the same underlying immersion as $i$, but with the $(x+1)$-th designated CE of $i$ now considered as nondesignated, and denoted $p_{n+1}$, and where $\tilde{\mathfrak{T}}$ is the temporary coorientation for $\tilde{i}$ which coincides with $\mathfrak{T}$ on $p_{1}, \ldots, p_{n}$ and which assigns to $p_{n+1}$ the coorientation it had as a designated CE of $i$. We continue discussing $H_{0}^{1}$ but clearly all will be true for $Q_{0}^{2}$ as well. By our assumption and Lemma 5.2 there exists $G \in V_{n-1}$ such that $f^{H}(i)=G\left(i^{\prime}\right)$ for all $i \in I_{0}^{x+1, y}$. Now let $J_{t}: F \rightarrow \mathbb{R}^{3}(0 \leq t \leq 1)$ be an AB equivalence as in the assumption of the lemma, between $i, j \in I_{0}^{x, y}$, so $J_{0}=i, J_{1}=j$, and assume the two additional CEs are passed at times $\frac{1}{3}$ and $\frac{2}{3}$. We make $J_{1 / 3}$ and $J_{2 / 3}$ into elements of $I_{0}^{x+1, y}$ by announcing the additional CE that is occurring as the $(x+1)$-th designated CE of type $H_{0}^{1}$. For $J_{1 / 3}$ we choose the coorientation of this $(x+1)$-th CE to be represented by the motion of $J_{t}$ through $J_{1 / 3}$ with increasing time, whereas for $J_{2 / 3}$ we use the motion of $J_{t}$ with decreasing time. 
So $J_{1 / 2}$ is on the positive side of both $J_{1 / 3}$ and $J_{2 / 3}$. The coorientation and order on all other designated CEs of $J_{1 / 3}$ and $J_{2 / 3}$ are those of $i$ which are continuously carried along the regular homotopy $J_{t}$. Similarly $J_{1 / 2}$ is made into an element of $I_{0}^{x, y}$ by continuously carrying the coorientation and order of the CEs of $i$ along $J_{t}$. We get

$$
f\left(J_{1 / 2}\right)-f(i)=f^{H}\left(J_{1 / 3}\right)=G\left(J_{1 / 3}^{\prime}\right)=G\left(J_{2 / 3}^{\prime}\right)=f^{H}\left(J_{2 / 3}\right)=f\left(J_{1 / 2}\right)-f(j) .
$$

The middle equality holds since $G$ is defined on $I_{0}$ and $J_{1 / 3}^{\prime}$ and $J_{2 / 3}^{\prime}$ are in the same connected component of $I_{0}$. And so we get $f(i)=f(j)$.

We now prove that $\mu_{n}\left(V_{n}^{x, y}\right) \subseteq E_{n}$, by induction on $n$. Assume it is true for any $k<n$ (and any $x, y$ ), so the conclusion of Lemma 5.3 holds. Let $i \in I_{n}^{x, y}$ have all its nondesignated CEs from the set $Y$ and located at $p_{1}, \ldots, p_{n}$ and let $\mathfrak{T}$ be a proper coorientation for $p_{1}, \ldots, p_{n}$. For $U, V \subseteq\left\{p_{1}, \ldots, p_{n}\right\}$ we will say $U \sim V$ if $U$ and $V$ include precisely the same points in $\left\{p_{1}, \ldots, p_{n}\right\}$ which are not of type $H_{0}^{1}$ or $Q_{0}^{2}$, the same number mod 2 of points of type $H_{0}^{1}$, and the same number mod 2 of points of type $Q_{0}^{2}$. It is easy to see that there are precisely $2^{r}$ sets in each $\sim$-equivalence class, where $r=r(C(i))$. If $U \sim V$ then there is an AB equivalence $J_{t}$ from $i_{\mathfrak{T}, U}$ to $i_{\mathfrak{T}, V}$ which passes an even number of CEs of type $H_{0}^{1}$ and then an even number of CEs of type $Q_{0}^{2}$, which we now construct. Starting with $i_{\mathfrak{T}, U}$, $J_{t}$ passes each CE in $U$ which is of type $H_{0}^{1}$, one by one, from the positive side determined by $\mathfrak{T}$, to the negative side, and then passes each CE in $V$ of type $H_{0}^{1}$ from the negative side to the positive side. $J_{t}$ then continues by doing the same for the CEs in $U$ and $V$ of type $Q_{0}^{2}$, finally arriving at $i_{\mathfrak{T}, V}$. And so by Lemma 5.3, for any $f \in V_{n}^{x, y}, f\left(i_{\mathfrak{T}, U}\right)=f\left(i_{\mathfrak{T}, V}\right)$. Since also $|U|=|V| \bmod 2$, we have

$$
(-1)^{n-|U|} f\left(i_{\mathfrak{T}, U}\right)=(-1)^{n-|V|} f\left(i_{\mathfrak{T}, V}\right) .
$$

Representatives for the various $\sim$-equivalence classes may be obtained as follows: Let $R \subseteq\left\{p_{1}, \ldots, p_{n}\right\}$ be a subset which includes all the points which are not of type $H_{0}^{1}$ and $Q_{0}^{2}$, and includes only one of the points of type $H_{0}^{1}$ if such exists, and only one of the points of type $Q_{0}^{2}$ if such exists. Then the set of subsets of $R$ includes precisely one representative from each $\sim$-equivalence class, and so we get:

Lemma 5.4. Let $i \in I_{n}^{x, y}$ have all its nondesignated CEs from the set $Y$ and located at $p_{1}, \ldots, p_{n}$ and let $r=r(C(i))$. Let $\mathfrak{T}$ be a proper coorientation for $p_{1}, \ldots, p_{n}$ and let $R \subseteq\left\{p_{1}, \ldots, p_{n}\right\}$ be as above, then for any $f \in V_{n}^{x, y}$ :

$$
\hat{f}(i)=f^{\mathfrak{T}}(i)=2^{r} \sum_{A \subseteq R}(-1)^{n-|A|} f\left(i_{\mathfrak{T}, A}\right) .
$$

This proves that $\mu_{n}(f)$ satisfies property (2) of Definition 3.1. 
As for property (1) of Definition 3.1, for given symbols $Z_{4}, \ldots, Z_{n}$ in $Y$, take $i \in I_{n+1}^{x, y}$ having its nondesignated CEs located at $\left\{p_{1}, \ldots, p_{n+1}\right\}$ and having

$$
\begin{aligned}
& C_{p_{1}}(i)=C_{p_{2}}(i)=H_{0}^{1}, \\
& C_{p_{n}}(i)=C_{p_{n+1}}(i)=Q_{0}^{2}, \\
& C_{p_{j}}(i)=Z_{j+1} \quad \text { for } j=3, \ldots, n-1 .
\end{aligned}
$$

Given a proper coorientation $\mathfrak{T}$ for $i$ at $p_{1}, \ldots, p_{n+1}$, let $i_{0} \in I_{n}^{x, y}$ be the immersion obtained from $i$ by resolving the $\mathrm{CE}$ at $p_{n+1}$ into the negative side determined by $\mathfrak{T}$, and let $i_{1} \in I_{n}^{x, y}$ be similarly defined using the point $p_{1}$. So the CEs of $i_{0}$ are $\left\{p_{1}, \ldots, p_{n}\right\}$ and those of $i_{1}$ are $\left\{p_{2}, \ldots, p_{n+1}\right\}$. Let $\mathfrak{T}_{0}, \mathfrak{T}_{1}$ be the proper coorientations for $i_{0}, i_{1}$ respectively, which are the restrictions of $\mathfrak{T}$, then for any $A \subseteq\left\{p_{2}, \ldots, p_{n}\right\},\left(i_{0}\right)_{\mathfrak{T}_{0}, A}=i_{\mathfrak{T}, A}=\left(i_{1}\right)_{\mathfrak{T}_{1}, A}$. Let $R \subseteq\left\{p_{2}, \ldots, p_{n}\right\}$ be the set including $p_{2}, p_{n}$ and all points which are not of type $H_{0}^{1}$ or $Q_{0}^{2}$. Then this $R$ may be used as the $R$ appearing in Lemma 5.4, for both $i_{0}$ and $i_{1}$. Furthermore $r\left(C\left(i_{0}\right)\right)=r\left(C\left(i_{1}\right)\right)$ which we denote $r$, so we get by Lemma 5.4:

$$
\hat{f}\left(i_{0}\right)=f^{\mathfrak{T}_{0}}\left(i_{0}\right)=2^{r} \sum_{A \subseteq R}(-1)^{n-|A|} f\left(i_{\mathfrak{T}, A}\right)=f^{\mathfrak{T}_{1}}\left(i_{1}\right)=\hat{f}\left(i_{1}\right) .
$$

Since $C\left(i_{0}\right)=\left[H_{0}^{1}, H_{0}^{1}, Q_{0}^{2}, Z_{4}, \ldots, Z_{n}\right]$ and $C\left(i_{1}\right)=\left[H_{0}^{1}, Q_{0}^{2}, Q_{0}^{2}, Z_{4}, \ldots, Z_{n}\right]$, this shows $\mu_{n}(f)$ satisfies property (1) of Definition 3.1. We have thus established $\mu_{n}\left(V_{n}\right) \subseteq E_{n}$, and so together with Section 4 we have $\mu_{n}\left(V_{n}\right)=E_{n}$.

To complete the proof of Theorem 3.2 it remains to show that given $f \in V_{n}(\mathbb{G})$ there is a function $s: L_{1} \rightarrow \mathbb{G}$ such that $f=s \circ f_{1}^{U}$. Since $\mu_{n}(f) \in E_{n}(\mathbb{G})$, there exists a homomorphism $\varphi: M_{n} \rightarrow \mathbb{G}$ such that $\varphi \circ g_{n}^{U}=\mu_{n}(f)$, and so

$$
\mu_{n}\left(\varphi \circ f_{n}^{U}\right)=\varphi \circ \mu_{n}\left(f_{n}^{U}\right)=\varphi \circ g_{n}^{U}=\mu_{n}(f),
$$

which is equivalent to $f-\varphi \circ f_{n}^{U} \in V_{n-1}(\mathbb{G})$. By induction on $n$ there is $\tilde{s}: L_{1} \rightarrow \mathbb{G}$ with $f-\varphi \circ f_{n}^{U}=\tilde{s} \circ f_{1}^{U}$ so $f=\varphi \circ f_{n}^{U}+\tilde{s} \circ f_{1}^{U}=\varphi \circ \pi_{n} \circ \mathscr{F} \circ f_{1}^{U}+\tilde{s} \circ f_{1}^{U}$ and we may take $s=\varphi \circ \pi_{n} \circ \mathscr{F}+\tilde{s}$.

\section{References}

[Goryunov 1997] V. V. Goryunov, "Local invariants of mappings of surfaces into three-space", pp. 223-255 in The Arnold-Gelfand mathematical seminars: geometry and singularity theory, edited by V. I. Arnold et al., Birkhäuser, Boston, 1997. MR 97m:57040 Zbl 0887.58007

[Max and Banchoff 1981] N. Max and T. Banchoff, "Every sphere eversion has a quadruple point", pp. 191-209 in Contributions to analysis and geometry (Baltimore, 1980), edited by D. N. Clark et al., Johns Hopkins Univ. Press, Baltimore, 1981. MR 83g:57020 Zbl 0544.57012

[Nowik 2000] T. Nowik, "Quadruple points of regular homotopies of surfaces in 3-manifolds", Topology 39:5 (2000), 1069-1088. MR 2001i:57033 Zbl 0962.57015 
[Nowik 2001a] T. Nowik, "Automorphisms and embeddings of surfaces and quadruple points of regular homotopies”, J. Differential Geom. 58:3 (2001), 421-455. MR 2003i:57034 Zbl 1032.57030

[Nowik 2001b] T. Nowik, "Finite order $q$-invariants of immersions of surfaces into 3-space", Math. Z. 236:2 (2001), 215-221. MR 2002a:57023 Zbl 0966.57025

[Nowik 2004] T. Nowik, "Order one invariants of immersions of surfaces into 3-space", Math. Ann. 328:1-2 (2004), 261-283. MR 2004m:57025 Zbl 1053.57021

[Nowik $\geq 2006]$ T. Nowik, "Formulae for order one invariants of immersions of surfaces", $A d v$. Math., to appear.

Received June 23, 2004. Revised December 15, 2004.

TAHL NOWIK

DEPARTMENT OF MATHEMATICS

BAR-ILAN UNIVERSITY

RAMAT-GAN 52900

ISRAEL

tahl@math.biu.ac.il

http://www.math.biu.ac.il/ tahl 